\title{
La lógica emocional y afectiva en la investigación educativa
}

\section{The logic emotional and affective in educational research}

Derling José Mendoza Velazco

Universidad Iberoamericana del Ecuador, Ecuador

Autor para correspondencia: derling969@gmail.com

Fecha de recepción: 01 de agosto de 2018 - Fecha de aceptación: 01 de diciembre de 2018

\section{Resumen}

El empleo de las tecnologías de información y comunicación en la educación universitaria motivó la presente investigación, cuyo objetivo consistió en elaborar una aproximación teórica de la lógica emocional y afectiva en la investigación educativa. La naturaleza de la investigación se desarrolló bajo el enfoque de cualitativo, sustentándose en el paradigma interpretativo-humanista. Por su esquema cualitativo, la Universidad Iberoamericana del Ecuador UNIB.E cuenta con una población de 650 estudiantes, de los cuales se aplicó la selección de muestra tipo homogénea, obteniendo la participación de diez (10) jóvenes. Se utilizó como instrumentos para la recolección de información, la guía de observación participativa y la entrevista de tipo semi-estructurada, los resultados fueron analizados a través de la triangulación generalizada de datos y teorías a través del software analítico del Atlas.Ti, donde se concluyó con una aproximación teórica formada por tres grandes constructos: Influencias cognoscitivas de las tecnologías de información y comunicación (TIC). Lógica emocional y Vínculos afectivos.

Palabras claves: educación; investigación; emociones; afecto; tecnologías de información y comunicación

\begin{abstract}
The use of information and communication technologies in university education motivated the present investigation, whose objective was to elaborate a theoretical approach of emotional and affective logic in educational research. The nature of the research was developed under the qualitative approach, based on the interpretative-humanist paradigm. Due to its qualitative scheme, Iberoamerican University of Ecuador UNIB.E has a population of 650 students, of which the homogeneous sample selection was applied, obtaining the participation of ten (10) young people. The participatory observation guide and the semi-structured interview were used as tools for information gathering, the results were analyzed through the generalized triangulation of data and theories through the Atlas. Ti analytical software, where concluded with a theoretical approach formed by three major constructs: Cognitive influences of information and communication technologies (TIC). Emotional logic and affective bonds.
\end{abstract}

Key words: education; research; emotions; affection; information and communication technologies 


\section{Introducción}

La motivación por estudiar las emociones en la educación con la aplicación de las TIC refleja, en gran medida, la utilidad para los educadores y especialistas, la influencia de la afectividad sobre el aprendizaje. Tradicionalmente, las investigaciones sobre la enseñanza y aprendizaje en los estudiantes, es abordada desde el punto de vista cognitivo, desertando el afecto del estudiante. Al respecto, el empleo de la tecnología, permite explicar algunas situaciones afectivas y emocionales que reprimen al estudiante confrontar con éxito la investigación.

En las actividades de aula, se aprecia ante las evaluaciones, la inseguridad y el bajo interés del estudio investigativo, como elementos que limitan un aprendizaje apropiado. La apreciación y valoración de las emociones y afectos en la educación, proporcionan nuevos modelos de procesar la enseñanza y aprendizaje, al introducir cambios en la metodología docente para superar las dificultades en su andragogía. Las creencias y atribuciones que reflejan los estudiantes universitarios, cultivan el predominio sobre los triunfos y fracasos de su carrera.

Considerándose un elemento indispensable en la sociedad educativa actual, dado que los conocimientos se están renovando constantemente con una asombrosa y vertiginosa velocidad. Se consideró necesario, analizar de manera exhaustiva, la incidencia de las tecnologías educativas y su perspectiva social, como objetivo principal de la investigación en realizar una aproximación teórica de la lógica emocional y afectiva de la investigación educativa para comprender y promover mediante las tendencias innovadoras de las TIC, las influencias cognoscitivas en las investigaciones de la UNIB.E.

\section{Fundamentación Teórica}

\section{El paradigma humanista}

(Rogers, 1961, pp. 6), como principal precursor de la teoría del humanismo expresa que "la conducta humana del hombre es exquisitamente racional, se mueve con ordenada y perspicaz complejidad hacia las metas que su organismo se esfuerza alcanzar". Destacándose la teoría en relación al hombre con características inherentes a su especie, describiendo lo positivo, al moverse o dirigirse hacia adelante, en base a lo constructivo, realista y digno de confianza, lo que tiende a la visión del desarrollo, sensible, responsivo, creativo y adaptable.

El investigador en base a la teoría humanista impugna las teorías conductistas de la educación, para presentar una visión diferente del ser, en una forma libre abierta e integral, y poder cumplir el episteme educativo. Ayudar a desarrollar la individualidad de las personas, apoyar a los alumnos a que se reconozcan como seres humanos únicos y asistir a los estudiantes a desarrollar sus potencialidades (Hamachek, 1987, pp. 171). Su objetivo entre el saber y la creencia, es promover el desarrollo del conocimiento investigativo de los estudiantes, como entes únicos que no solo participan cognitivamente en las aulas, sino como individuos que ostentan del afecto. 


\section{La inteligencia emocional}

La inteligencia emocional como segundo sustento teórico, es definida (Salovey y Mayer, 1997, pp. 189) como: “La habilidad para percibir, valorar y expresar emociones con exactitud, la habilidad para acceder y/o generar sentimientos que faciliten el pensamiento; la habilidad para comprender emociones y el conocimiento emocional y la habilidad para regular las emociones proviniendo un crecimiento emocional e intelectual".

La destreza para tomar el control y tener comprensión de las emociones y sentimientos, propios y ajenos, es puntualizada como la inteligencia emocional, que ayuda a motivar y empeñar las posibles frustraciones, que nacen en la alteración de los impulsos, de la misma manera los estados de ánimo, al disminuir la angustia, que interfiere en la preponderancia de la racionalidad y confianza con los demás individuos.

Igualmente, para (Goleman, 1999, pp. 5), la inteligencia emocional es poseer "la capacidad de reconocer los sentimientos propios y ajenos, de poder auto motivarse para mejorar positivamente las emociones internas y las relaciones con los demás". Este autor, muestra que la inteligencia emocional accede la conciencia de los sentimientos facilitados en el momento en el que se aprecian, facilitando una atención gradual y continua a los propios cambios internos. En esta conciencia auto reflexiva, la mente del ser humano, visualiza y estudia las prácticas mismas; incluidas las del contorno de las emociones.

Por otra parte, (Ryback, 2008, pp. 325), hace mención a que:

"La mejor manera de definir la inteligencia emocional es considerar que se trata de la capacidad de aplicar la conciencia y la sensibilidad para discernir los sentimientos y subyacentes de la comunicación interpersonal, para resistir la tentación que mueve a reaccionar de una manera impulsiva irreflexiva, obrando en vez de ello con receptividad, autenticidad y sinceridad".

En efecto, el manejo adecuado de la inteligencia emocional por parte del investigador y el estudiantado, les va permitir actuar con empatía, utilizando la comprensión en pro de beneficiar a los estudiantes a su cargo y transformar el espacio educativo en un status de armonía y respeto, donde no se presente dificultades en las actividades de investigación por la falta de apoyo o por actuar descontroladamente ante situaciones que no ameritan tanta atención.

\section{El paradigma Interpretativo}

Los paradigmas de estudio facilitan la ubicación y conocimiento del investigador, para comprender mejor los fenómenos que se van desarrollando para desencadenar propuestas de mejora permanente dentro del contexto de estudio, en este sentido para (Ricoy 2006, pp. 14), “el paradigma interpretativo se considera interpretativo-simbólico, cualitativo, naturalista, humanista y fenomenológico". Según las variables conceptuales del autor el paradigma interpretativo se presenta como una alternativa amplia en el campo de la educación, presentando sus antecedentes históricos en la fenomenología, el interaccionismo simbólico interpretativo y el humanismo. 
En base a la investigación de carácter cualitativo se caracteriza el paradigma interpretativo en la búsqueda y profundización de la comprensión lógica y actitudinal de los estudiantes ligado fundamentalmente en el escenario, en especial en el contexto investigativo, contribuyendo a la intuición y el conocimiento para actuar frente a otras situaciones similares. El estudio de situaciones naturales con seres humanos precisa de un encuadre metodológico congruente con tal particularidad. El estudio se basa en este paradigma para visualizar en hondura la situación aprendida y aportar nuevos descubrimientos a la comunidad educativa.

\section{El interaccionismo simbólico}

Como componente conductual del paradigma interpretativo relaciona los aspectos universales, psicológicos y sociológicos en el ser humano, su principio teórico se encuentra establecido al indicar que, la capacidad de pensamiento esta modelada por la interacción social (Blumer, 1969, pp. 271).

De manera general (Shott, 1979, pp. 47), indica que "las estructuras sociales y las regulaciones normativas son el marco de la acción más que su determinante y modelan la conducta sin dictarla ineluctablemente". La interacción simbólica postula el trabajo cognitivo y emocional afirmando que la construcción de emociones es maleable y moldeable por las influencias sociales. En el interior de los límites impuestos por las normas sociales y por los estados internos, los individuos construyen sus emociones, las definiciones e interpretaciones que realizan son centrales en este proceso investigativo.

En este sentido, para el desarrollo de la investigación el interaccionismo simbólico asume que las expresiones internas y externas son una producción socio educativa, ello no indica que las alocuciones se improvisen de situación en situación, sino que el ambiente orientativo establece las normas de las expresiones, del sentimiento adecuado a la definición de las situaciones, y que poco a poco se van instaurando en los estudiantes objetivando en las relaciones personales del estudiantado en las investigaciones mediante uso de las TIC. De esta manera el status educativo organizado por el docente investigador será quien establezca los ideales, patrones, valores y normas que encausan y objetivan el componente conductual de las expresiones, y quien proponga a los estudiantes de que, como y cuando se apliquen las actividades de investigación, así como el significado de su experiencia, y no solo de su conducta.

\section{Metodología}

En virtud de la metodología se consideró pertinente realizar la investigación dentro del enfoque cualitativo caracterizado cuando se busca, en comprender los fenómenos, explorándolos desde la perspectiva de los participantes en un ambiente natural y en relación con su contexto (Hernández, Fernández y Baptista, 2014, pp. 358).

Desde el mismo ámbito investigativo el estudio se sustenta el método cualitativo caracterizado por (Hernández y otros, 2017, pp. 114) cuando:

"El investigador quiere explorar, describir y co-nocer con amplitud y profundidad percepciones, emociones, sentimientos, experiencias, enfoques y puntos de vista de personas, 
desde la perspectiva de los propios participantes o sujetos investigados, en su ambiente natural y de manera más abierta".

El investigador al formar parte de la plataforma investigativa de la UNIB.E, le permite percibir, estructurar y relacionar las actividades investigativas de los estudiantes con la aplicación de las TIC a fin de dar respuesta a los problemas presentados, donde se establecieron relaciones entre la teoría y las prácticas tecnológicas, para generar premisas y criterios en el contexto emocional y afectivo de los estudiantes.

\section{Población y Muestra}

En el estudio, se aplicó la selección de informantes como indica (Rojas, 2007, pp. 76) "muestreo de tipo homogéneo: el cual tiene como propósito describir un grupo específico en profundidad". La UNIB.E cuenta en su totalidad con 650 estudiantes, de los cuales aplicando el muestreo tipo homogéneo se seleccionaron 10 informantes de distintos niveles y carreras. Los estudiantes como conjuntos semejantes en la universidad, participaron en el progreso y desarrollo del objeto de estudio presentando su disponibilidad, para la observación y análisis de sus capacidades cognitivas, como también la manipulación y conocimiento de las TIC.

En síntesis, para la escogencia de los informantes, se elaboró un taller con preguntas sencillas y comprensibles dándose a demostrar, la solicitud de la participación. Para la selección se aplicó la técnica de grupos focales, definido por (Aigneren, 2002, pp. 7) como "una metodología de talleres o reuniones con un grupo escogido de individuos con el objetivo de obtener información acerca de sus puntos de vista y experiencias sobre hechos, expectativas y conocimientos de un tema. El propósito de la actividad, fue seleccionar los estudiantes como grupo representativo y participativo, que facilitaron información relevante y necesaria para la recolección de información.

\section{Análisis de los datos}

La recolección de datos implicó técnicas e instrumentos, que fueron utilizados para desarrollar los sistemas de información de la investigación y así enfocar la verificación del problema planteado, aplicándose la guía de observación, donde docente investigador participo en las dinámicas cotidianas y se unió, al interactuar visualizar y captar, en forma sistemática, todo hecho situación o fenómeno presentado por los estudiantes en la aplicación de las TIC, en sus prácticas investigativas. De igual forma se desarrolló la entrevista de tipo semiestructurada, permitiendo una mayor libertad y flexibilidad en la obtención de la información, facilitando una base informativa compleja. Los datos obtenidos fueron analizados, mediante la contrastación teórica y el método de comparación continua a través del sistema operativo Atlas.Ti versión actual proporcionando la categorización de los resultados.

\section{Categorización}

Después de haberse transcrito toda la información, se procedió a una revisión y organización mediante criterios y discernimientos de lógica analítica, se agregó la información de recabado de datos, aunando los textos de la entrevista semi-estructurada y de la observación 
participativa moderada, en el software Atlas.ti versión 7.5.4, donde se formaron las categorías acertadas, descritas por (Strauss y Corbin, 2002, pp. 124) como "conceptos derivados de los datos, que representan los fenómenos". Los conceptos y temas emanados de la investigación determinaron las unidades de análisis, para tener una descripción más precisa de los resultados, apreciándose que la abultada información se minimizo en partes, para proporcionar una mayor intuición del material analizado, codificándose en solo elementos con ítems representativos de los instrumentos a aplicar, que después se clasificaron y agruparon, facilitando la búsqueda de posibles vinculaciones o relaciones para obtener la aproximación teórica del objeto de estudio.

\section{Resultados}

De acuerdo a la información conseguida en las observaciones y entrevistas con los estudiantes, en respuesta a los objetivos de estudio, se tomaron las categorizaciones como constructos para la aproximación teórica, entre las cuales se encuentran, influencias cognoscitivas de las TIC, la lógica emocional y los vínculos afectivos. A continuación, se presentan los constructos de acuerdo con cada categoría:

\section{Categoría 1. Influencias cognoscitivas de las TIC}

Los resultados del estudio, demostraron que las corrientes psicológicas cognoscitivas fomentadas con la aplicación de las TIC, alcanzan el predominio en el campo investigativo del aprendizaje humano, acumulando evidencias suficientes, para afirmar que la mayoría de los estudiantes necesitan laborar con modelos y representaciones digitales, para un aprendizaje significativo de la investigación (figura 1).

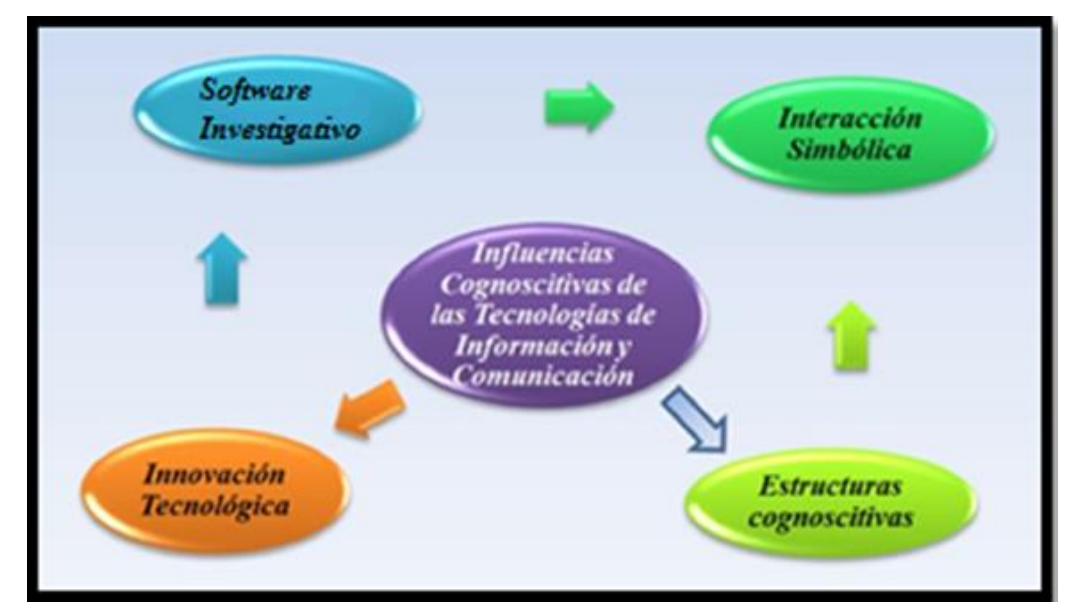

Figura 1. Influencias cognoscitivas de las tecnologías de información y comunicación

Así pues, la mayoría de los estudiantes en la búsqueda de información compleja para sus estudios, se demanda el talento de los participantes en el uso de las TIC, donde los contenidos al ser expuestos en las redes, debe ofrecer a los estudiantes oportunidades para que diseñen planes de acción para ser abordados; discusión de la idoneidad y viabilidad de las acciones planeadas; examinación continua de sus posibilidades reales de ponerlas en juego; formulación de preguntas y cuestionamientos, organización estratégica de los recursos (cognitivos, materiales, documentales, tecnológicos) de los que se dispone para llevar a cabo la tarea resolutoria, donde 
el joven se siente parte de las actividades de búsqueda de datos y no solo receptor de información.

Por otra parte, las TIC privilegian el trabajo en equipo, pues éste no sólo permite el desarrollo de habilidades cognoscitivas, sino, además, de una manera muy efectiva de aprender y así poder visualizar las cualidades que las TIC permiten al avanzar de forma significativo en el razonamiento lógico de organización, mediante la comunicación con otros compañeros. Lo fundamental de la tecnología se relaciona con su potencial de interacción, de ser interfaz para construcción del saber, donde la imaginación e intervención del sujeto varía constantemente, además de distinguirse las actitudes del interaccionismo simbólico, estas presentes entre la imagen y la imaginación, de adaptación de los estudiantes.

\section{Categoría 2. La lógica emocional}

Para comprender la lógica emocional (Aimelet y Massot, 2015, pp. 1) describen que: "Estamos copilotos. A través de nuestro cerebro "reptiliano", automático y rápido, que rige nuestra relación inmediata con los demás y con el medio ambiente. Y nuestro cerebro "cognitivo", consciente pero más lento, lo que da sentido y permite dar un paso atrás. El acuerdo entre los dos copilotos no siempre es cordial, ni mucho menos. Su discordia crea estrés. Este es el diálogo interno, una fuente de presión emocional constante, que nuestro parásito sentimientos y degrada nuestra relación, que "la lógica emocional" permite descifrar.

Es por ello, que la inteligencia emocional, se conceptualiza como la destreza que posee el individuo para percibir, conocer, valorar y expresar emociones con la misma habilidad para acceder a ella, facilitando el pensamiento, de tipo comprensivo, para regular las emociones promoviendo un desarrollo emocional e intelectual.

Al estudiar los resultados cualitativos referentes a los aspectos emocionales, la lógica emocional como categoría en el presente estudio, puede definirse como el sentido que conduce las emociones y estado de ánimo, es decir; el impulso que conduce al estudiante a actuar o reaccionar de forma lógica en determinadas circunstancias de su investigación educativa (figura 2).

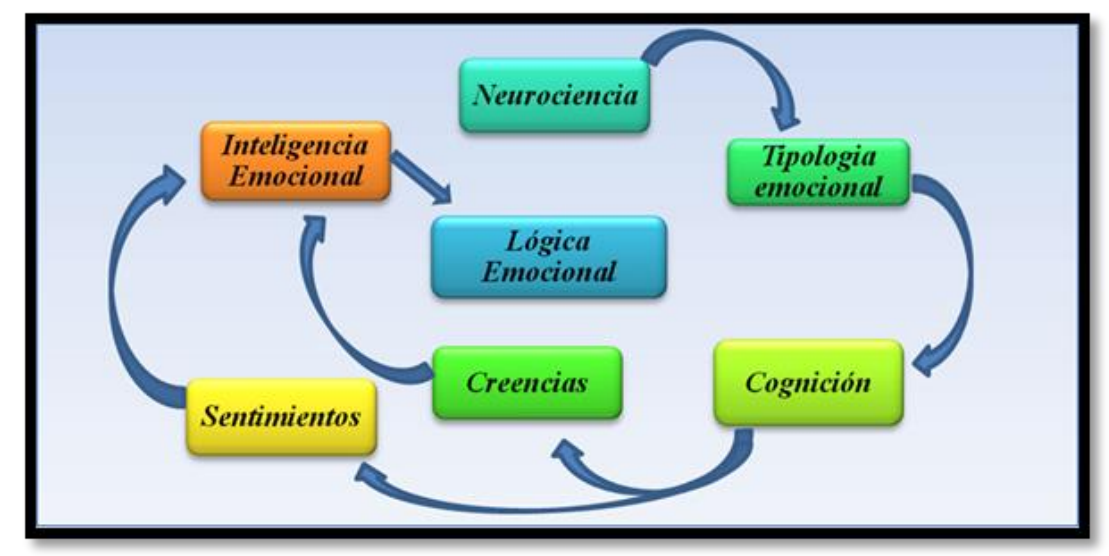

Figura 2. Lógica emocional 


\section{Categoría 3. Los vínculos afectivos}

En los datos analizados del estudio, se percibieron los contagios emocionales al destacar la empatía y las actitudes de los estudiantes que aportan un impacto positivo en las actividades. El investigador al igual considera, que los jóvenes demostraron, confianza y motivación con un estilo de aprendizaje perceptual sensitivo, para acceder a la información con mayor facilidad, fortaleciéndose el dominio afectivo definido por (McLeod, 1989, pp. 245) como "un extenso rango de sentimientos y humores (estados de ánimo) que son generalmente considerados como algo diferente de la pura cognición e incluye como componentes específicos de este dominio las actitudes, creencias y emociones".

Atendiendo al proceso cognitivo sobre el proceso de investigación educativa, la incorporación de la TIC, favorece la participación activa y efectiva de los estudiantes, al fomentar los componentes afectivos, percepción, inteligencia, memoria y pensamiento de los mismos. Estas situaciones de incursión y diversidad tecnológica pueden determinar el afecto de la educación universitaria en los estudiantes.

Los vínculos afectivos, son el enlace emocional que emerge entre la aplicabilidad de las TIC y la investigación universitaria. Este vínculo se debe conformar por diferentes aspectos relevantes de las actitudes estudiantiles que son conformadas, por los principios socios educativos y familiares, donde la investigación deja de ser exacta, rigurosa y fría para convertirse en un área de conocimiento afectivo. Para introducir los estudiantes en los vínculos afectivos, se debe emprender la dotación y conocimientos, del dominio afectivo, ya que no solo se puede establecer un aprendizaje por medio de los alcances cognitivos, procedente de la meta cognición también debita considerarse la dimensión afectiva del estudiante.

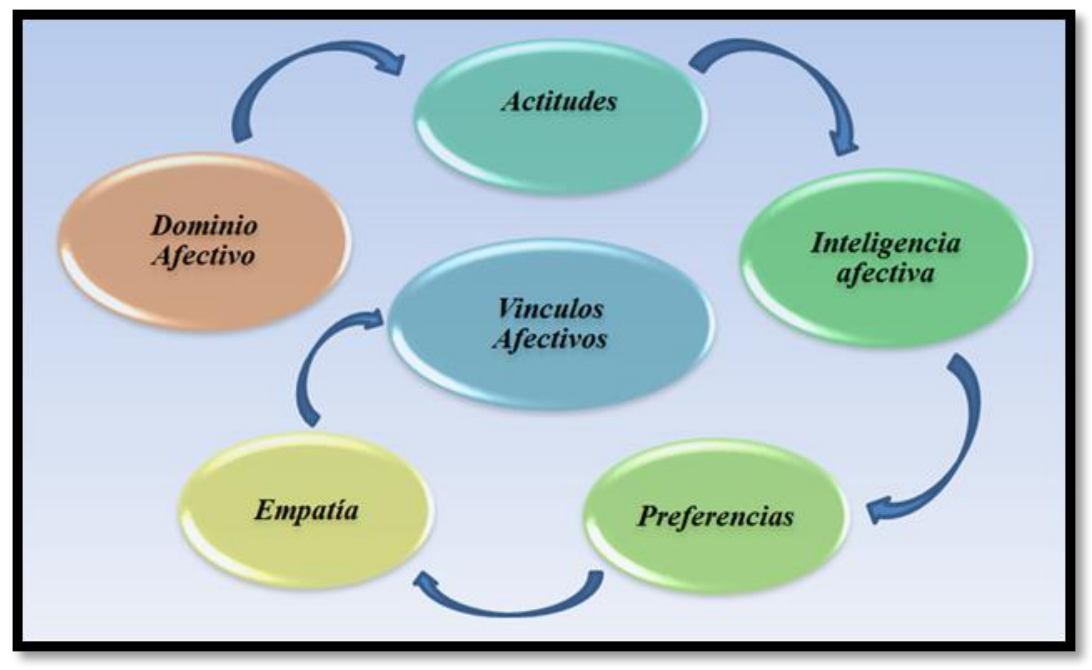

Figura 3. Los vínculos afectivos

Es así, que se pone de manifiesto que el cuestionamiento afectivo, mantiene un papel importante en la educación matemática, estando fuertemente arraigado a los estudiantes sin darse cuenta muchas veces el poseer sentimientos, sin poder conocerlos o controlarlos. El dominio afectivo (Mendoza, 2016, pp. 252), lo conceptualiza como "los elementos que conforman el afecto de manera general, puesto que también saber considerarlos y manipularlos, entre estos se 
distinguen, los gustos, la confianza, el comportamiento, las emociones, las creencias las preferencias y las relaciones afectivas".

\section{Conclusiones}

Al realizar la contrastación de las categorías se puede concluir que la Inteligencia Emocional ha sido objeto de múltiples atribuciones de éxito en la vida profesional e investigativa en los estudiantes. Algunas instituciones laborales, la han retomado e incluyen como característica deseable de los empleados. Así mismo, en universidades donde ha llegado a ser considerada como un ideal a implementar la Inteligencia Cognitiva, favoreciendo el desarrollo integral del estudiante.

El investigador resalta que la Inteligencia Emocional es más importante que la Inteligencia Cognitiva para el éxito del individuo en su vida cotidiana, laboral e investigativa. Sin embargo, la evidencia empírica ha sido clara al respecto, debido a los resultados investigativos. Por un lado, se encuentran docentes que ameritan la formación educativa emocional como un buen predictor de éxito y que está relacionado con el buen rendimiento o desempeño de los estudiantes en la UNIB.E. Por otro lado, la universidad requiere de docentes que opten por las cualidades del conocer tecnológico que demuestre relaciones significativas con el éxito investigativo.

Al complementar los elementos emergentes distinguidos como las categorías, con la debida sustentación teórica, así como los datos cualitativos contribuidos por los estudiantes que participaron en las actividades investigativas en la UNIB.E finalmente surge una aproximación teórica, donde la interacción que el estudiante ejerce con el medio tecnológico facilita su capacidad de pensamiento investigativo, por permitirle trabajar en forma sencilla y accesible; donde las emociones cumplen una función vinculable a su entorno actualizable y moderno.

\section{Bibliografía}

Aigneren, M. (2002). La técnica de recolección de información mediante los grupos focales. Universidad de Antioquia. Colombia. Disponible: http://aprendeenlinea.udea.edu.co/revistas/index.php/ce o/art icle/v iewFile/1611/1264

Aimelet, C. y Massot, P. (2015). E.m.o.t.i.o.n. 7 étapes pour se comprendre. Francia: Albin Michel.

Blumer, H. (1969). Symbolic Interactionism. Perspective and Method, Berkeley and Los Angeles, California, University of California Press.

Goleman, D. (199). La Inteligencia Emocional. Barcelona: Kairós

Hamachek, D. (1987). Encounters with the self. New York. Holt: Rinehart \& Wiston.

Hernández, R. Fernández, C, y Baptista, P. (2014). Metodología de la investigación. Mèxico: McGraw-Hill Interamericana. 
Hernández, R. Mendez, S. Mendoza, C. y Cuevas A. (2017). Fundamentos de investigación. Mèxico: McGraw-Hill Interamericana.

McLeod, D. B. (1989). The role of affect in mathematical problema solving. New York: Springer Verlag.

Mendoza Velazco D. (2016). La matemática emocional y afectiva a partir del empleo de las tecnologías de información y comunicación en educación media general. Rubio: UPEL.

Ricoy Lorenzo, C. (2006). Contribución sobre los paradigmas de investigación. Educação. Revista do Centro de Educação, 31 (1), 11-22.

Rogers, C. (1961). El proceso de convertirse en persona: mi técnica terapeuta. Buenos Aires, Paidós.

Rojas de Escalona, B. (2007). Investigación Cualitativa. Caracas: Fedupel.

Ryback, D. (2008). Trabaje con su Inteligencia, los factores Emocionales al servicio de la Gestión Empresarial y el Liderazgo Efectivo. España: Editorial EDAF, S.A.

Salovey, P. y Mayer, J. (1997). What is emotional intelligence?. New York: Basic Books.

Shott, S. (1979). Emotion and social life: a symbolic interactionist analysis. American Journal of Sociology, 12- 84 .

Strauss, A. y Corbin, J. (2002). Bases de la investigación cualitativa. Técnicas y procedimientos para desarrollar la teoría fundamentada. Colombia: Universidad de Antioquia. 